\title{
Long-term outcomes of thigh arteriovenous graft stenting
}

\begin{abstract}
Background: Stents have been increasingly used for treating venous anastomosis stenosis seen in arteriovenous grafts (AVGs). A major reason for this trend is that stents can potentially confer a better patency rate compared to angioplasty. However, limited data are available about the outcomes of stents that are used to treat thigh AVG dysfunction. This study sought to assess the primary and secondary patency rates of stents used to treat thigh AVGs dysfunction at one year.

Methods: This is a retrospective study of dialysis patients who received therapy via thigh grafts $(\mathrm{N}=50)$ and underwent stent placement between January 2005 and June 2017 at our center. Data on demographics and baseline characteristics of the study population were collected. The primary and secondary patency rates were defined as the time between stent deployment and the first intervention and second intervention, respectively. Patency and re-intervention rates were estimated using Kaplan-Meier survival analysis.

Results: This study included 50 patients with thigh AVGs; mean age was $50.5 \pm 15.5$ years; $52 \%$ were female; $80 \%$ were black; and $90 \%$ had hypertension. The main indication for stenting was thrombosis due to venous anastomosis stenosis (74\%). The number (mean \pm SD) of stents deployed was $1.24 \pm 0.8$. The primary patency rate at three months and one year was $58.7 \%$ and $30.7 \%$. In comparison, the secondary patency rate at three months and one year was $68.2 \%$ and $40.7 \%(p=0.04)$.
\end{abstract}

Conclusions: Thigh AVG stenting can be successfully used to improve the overall patency rates of failing AVGs.

Keywords: dialysis, thigh, stent, arteriovenous graft, patency rate
Volume 9 Issue 3 - 202।

\author{
Masa Abaza BS,' Sloan E Almehmi, ${ }^{2}$ Alian Al- \\ Balas, ${ }^{3}$ Ammar Almehmi ${ }^{3}$ \\ 'University of Alaska at Anchorage, Biology Department, USA \\ ${ }^{2}$ University of Alabama at Birmingham, Biology Department, \\ USA \\ ${ }^{3}$ University of Alabama at Birmingham, Department of Radiology \\ and Medicine, USA
}

Correspondence: Ammar Almehmi, University of Alabama at Birmingham, Department of Radiology and Medicine, USA, Tel, (205) 975-4850, Fax (205) 975-5257,

Email aalmehmi@uabmc.edu

Received: August 24, 2021 | Published: September 27, 2021

\section{Introduction}

Arteriovenous thigh grafts (AVGs) have been utilized when the upper extremity vascular access options are exhausted. Similar to their counterparts of the upper extremity grafts, thigh AVGs are usually complicated with stenotic lesions at the venous anastomosis site that often leads to dialysis complications such as bleeding, elevated venous pressure, and eventually graft thrombosis. ${ }^{1}$ These complications are associated with poor survival of the dialysis access. Accordingly, catheter-based therapy, including balloon angioplasty and stenting, are developed to manage these complications. ${ }^{1,2}$ Limited data is available about the outcomes of thigh AVG stenting in dialysis patients. ${ }^{3}$ The overarching goal of this study was to report the primary and secondary patency rates among dialysis patients with AVGs.

\section{Methods}

We retrospectively reviewed the medical records of dialysis patients $(\mathrm{N}=50)$ who received dialysis via thigh AVGs and underwent stent placement between January 2005 and June 2017 at our center. Data on demographics and baseline characteristics of the study population were collected. The main covariates used in the analysis included age, race, gender, diabetes, hypertension, number of stents, stent location and stent laterality. Baseline characteristics of the study population were compared using chi-square tests for categorical values and t-tests for continuous variables.

The primary and secondary patency rates were defined as the time between stent deployment and the first intervention and second intervention, respectively. Kaplan-Meier survival analyses were used to calculate the time to first and second interventions.

\section{Results}

The baseline characteristics of the study population are shown (Table 1). The study cohort included 50 hemodialysis patients with a mean age of $( \pm \mathrm{SD}) 50.48(15.45)$ years. The number of stents ranged from 1 to 6 stents with a mean $( \pm \mathrm{SD})$ of $1.24(0.80)$. While the mean number of days until first intervention after stent placement was 250.95 (325.25), the mean time to the second intervention was 284 (599.17) days (range 3-3108). The technical success rate of stent deployment was $100 \%$ with no complications were encountered including stent migration, dislodge or fracture. The relationship between the main study covariates and the time to the first intervention revealed that white patients had a longer primary patency as compared to black patients $(p=0.012)$. There was no relationship between the study covariates and the time to the secondary intervention. The number of stents placed was significant in determining the primary patency with the shorter time to second intervention, the greater the number of stents placed ( $p=0.0149$ ). The survival curves of the dialysis access after stenting at 3,6, and 12 moths are shown (Figure 1). At 3 and 12 months, the primary and secondary patency rates were $41.3 \%$ vs $31.3 \%$ and $69.3 \%$ vs $59.3 \%$, respectively $(p=0.039)$. The type of stent used (covered versus uncovered) approached statistical significance $(p=0.063)$, which may indicate a trend towards improved primary patency with covered stents. 


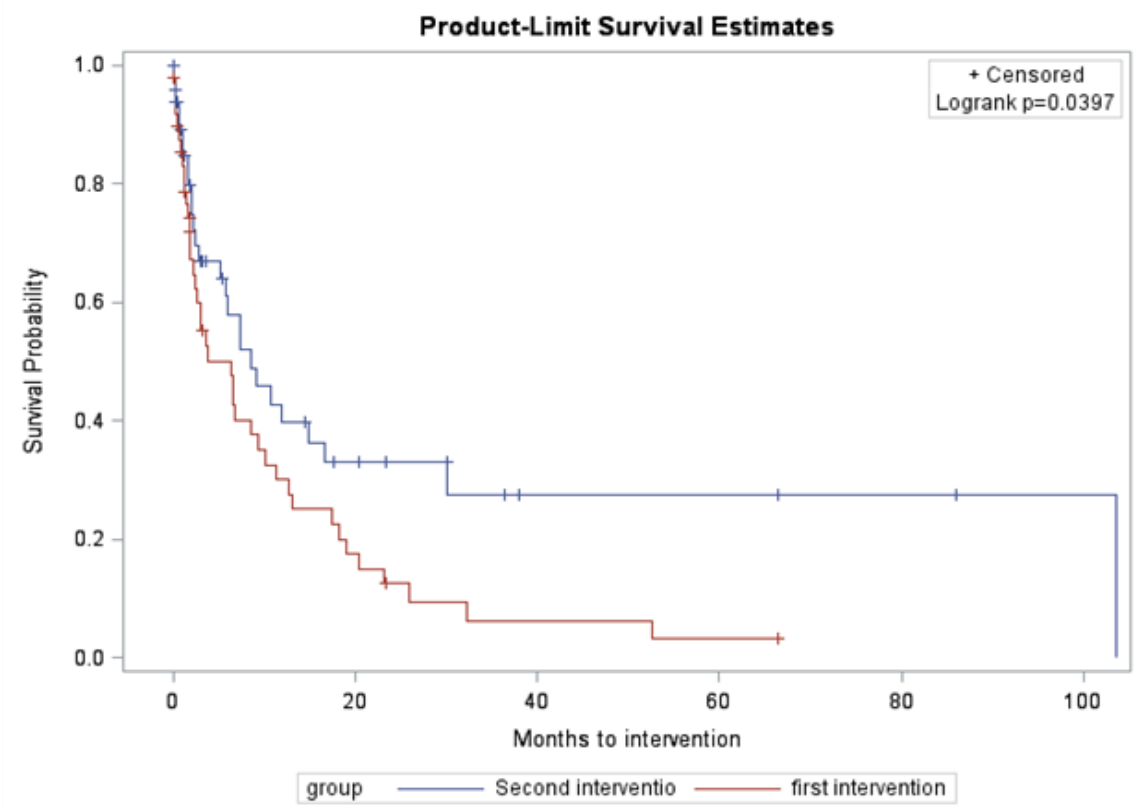

Figure I Kaplan-Meier curves showing primary and secondary patency rates.

Table I Baseline Characteristics of the study population

\begin{tabular}{ll}
\hline Characteristic & N (\%) \\
\hline Gender, Male & $24(48 \%)$ \\
Race, Black & $10(20 \%)$ \\
Diabetes, yes & $15(30 \%)$ \\
Hypertension, yes & $45(90 \%)$ \\
Stent laterality, left & $26(52 \%)$ \\
Stent type, covered & $20(40 \%)$ \\
Stent location, venous anastomosis & $37(74 \%)$
\end{tabular}

\section{Discussion}

Hemodialysis access continues to pose a major challenge in caring for patients with end-stage renal disease. In those patients who are not candidates for arteriovenous fistula creation, AVG is considered the next best option for vascular access. ${ }^{4}$ However, these AVGs are subject to multiple complications such as stenosis, infections, aneurysmal formations, and thrombosis. These complications are known to shorten the lifespan of these grafts, necessitating the search for new sites for creating dialysis access. ${ }^{5}$ It is well known that long term central venous catheters are associated with higher complications rate and increased mortality. Therefore, thigh AVGs became an attractive option which can be used as a catheter-sparing strategy in dialysis patients. As with other AVGs in other sites, these grafts are often complicated with inadequate venous outflow resulting in access thrombosis and failure. Ninety percent of AVG failures are caused by outflow stenosis at the venous anastomosis between the synthetic graft and the native vein. ${ }^{6}$

Catheter-based approaches (i.e., balloons, cutting balloons, brachytherapy) have been utilized to manage these complications with suboptimal outcomes. Six-month AVG patency rates of the upper extremity grafts after balloon angioplasty range between $20 \%$ and
$41 \% .^{5,7,8}$ In order to improve the long term AVG patency and decrease the interruptions in dialysis therapy, stent deployment at the target lesions were developed. It was hypothesized that covering the stent with graft material would decrease the in-stent restenosis related to the neointimal hyperplasia. ${ }^{5,6}$

In the current study, we reviewed the patency rate of thigh AVG stents that were used to treat venous outflow stenoses. We found that the overall patency rate of failing grafts had improved after stenting the venous anastomosis. We speculate that AVG stenting had overcome the recurrent intimal hyperplasia at the venous outflow and the resistance to balloon angioplasty. ${ }^{8,9}$

With regards to the longer primary patency rate among white patients, we speculate that this difference may be related to issues of access to health care. On the other hand, the increased number of stents was associated with shorter primary patency rate. This could be related to the presence of multiple lesions within the dialysis access reflecting its poor quality.

Our findings differ from previously published studies in that secondary patency was improved over primary patency in our patient population. It is worth mentioning that previous studies have mainly evaluated the stents of the upper extremity AVGs; the findings of the upper extremity AVG stent may not be extrapolated to their counterparts of the lower extremity.

While thigh AVG stents demonstrate poor primary patency rate at one year, the secondary patency rate is significantly better during the first year after stenting. Prospective randomized clinical trials are needed to further elucidate these findings.

Our study is limited by its retrospective nature and small sample size. Further prospective case control clinical trials are needed.

In conclusion, stenting of thigh grafts is a feasible option in managing dialysis access dysfunction related to circuit lesions and may prolong the life span of these accesses. 


\section{Funding}

The authors received no financial support for the research, authorship, and/or publication of this article.

\section{Declaration of conflicting interests}

The authors declared no potential conflicts of interest with respect to the research, authorship, and/or publication of this article.

\section{Author contributions}

All authors contributed equally to this article. All authors read and approved the final manuscript.

\section{References}

1. Haskal JZ, Trerotola S, Dolmatch B, et al. Stent Graft versus Balloon Angioplasty for Failing Dialysis-Access Grafts. $N$ Engl $J$ Med. 2010;362:494-503.

2. Karnabatidis D, Kitrou P, Spiliopoulos S, et al. Stent-grafts versus angioplasty and/or bare metal stents for failing arteriovenous grafts: a cross-over longitudinal study. J Nephrol. 2013;26(2):389-395.
3. Schmelter C, Raab U, Lazarus F, et al. Outcomes of AV Fistulas and AV Grafts after Interventional Stent-Graft Deployment in Haemodialysis Patients. Cardiovasc Intervent Radiol. 2015;38(4):878-886.

4. Schild AF. Maintaining vascular access: the management of hemodialysis arteriovenous grafts. J Vasc Access. 2010;11(2):92-99.

5. Webb KM, Cull DL, Carsten CG, et al. Outcome of the use of stent grafts to salvage failed arteriovenous accesses. Ann Vasc Surg. 2010;24(1):34-38.

6. Davila Santini L, Etkin Y, Nadelson AJ, et al. Stent-grafts improve secondary patency of failing hemodialysis grafts. $J$ Vasc Access. 2012;13(1):65-70.

7. Vesely TM, Siegel JB. Use of the peripheral cutting balloon to treat hemodialysis-related stenoses. J Vasc Interv Radiol. 2005;1593-1603.

8. Vogel PM, Parise C. SMART Stent for salvage of hemodialysis access grafts. J Vasc Interv Radiol. 2004;15:1051-1060.

9. Mousa AY, Patterson W, Abu-Halimah S, et al. Patency in arteriovenous grafts in hemodialysis patients. Vasc Endovascular Surg. 2013;47(6):438-443. 